\title{
Hybridization of Cognitive Radar and Phased Array Radar Having Low Probability of Intercept Transmit Beamforming
}

\author{
Abdul Basit, ${ }^{1}$ Ijaz Mansoor Qureshi, ${ }^{2,3}$ Wasim Khan, ${ }^{1}$ \\ Ihsan Ulhaq, ${ }^{1}$ and Shafqat Ullah Khan ${ }^{4}$ \\ ${ }^{1}$ Department of Electronic Engineering, International Islamic University, H-10 Sector, Islamabad, Pakistan \\ ${ }^{2}$ Department of Electrical Engineering, Air University, Islamabad, Pakistan \\ ${ }^{3}$ Institute of Signals, Systems and Soft Computing (ISSS), Islamabad, Pakistan \\ ${ }^{4}$ Isra University, Islamabad Campus, Pakistan \\ Correspondence should be addressed to Abdul Basit; abdulbasit@iiu.edu.pk
}

Received 21 September 2013; Accepted 2 March 2014; Published 31 March 2014

Academic Editor: P. Mohanan

Copyright (C) 2014 Abdul Basit et al. This is an open access article distributed under the Creative Commons Attribution License, which permits unrestricted use, distribution, and reproduction in any medium, provided the original work is properly cited.

\begin{abstract}
A novel design of a cognitive radar (CR) hybridized with a phased array radar (PAR) having a low probability of intercept (LPI) transmit beam forming is proposed. PAR directed high gain property reveals its position to interceptors. Hence, the PAR high gain scanned beam patterns, over the entire surveillance region, are spoiled to get the series of low gain basis patterns. For unaffected array detection performance, these basis patterns are linearly combined to synthesize the high gain beam pattern in the desired direction using the set of weight. Genetic algorithm (GA) based evolutionary computing technique finds these weights offline and stores to memory. The emerging CR technology, having distinct properties (i.e., information feedback, memory, and processing at receiver and transmitter), is hybridized with PAR having LPI property. The proposed radar receiver estimates the interceptor range and the direction of arrival (DOA), using the extended Kalman filter (EKF) and the GA, respectively, and sends as feedback to transmitter. Selector block in transmitter gets appropriate weights from memory to synthesize the high gain beam pattern in accordance with the interceptor range and the direction. Simulations and the results validate the ability of the proposed radar.
\end{abstract}

\section{Introduction}

The research towards the radar/smart antenna that can adapt itself to the environment changes or with the unknown time varying scenarios has been one of the dynamic research areas [1]. The radar design technology has been dominated not only by signal processing and control techniques alone but also by the hybridization of both; it has met many milestones over five decades. These advancements in the field of radar design have addressed different types of problem such as range identification, radar cross section (RCS) measurement, speed estimation, and direction estimation, but the analogy between the human brain and the radar has given birth to the concept of the cognitive radar [2]. In the basic cognitive radar design, its transmitter changes properties/abilities according to the radar environment variations, based on the information feedback from its receiver, where the feedback works as a facilitator of intelligence [3]. A basic cognitive radar should be (i) continuously sensing its environment, (ii) electronically scanning the environment in fast manner, and (iii) utilizing the digital signal processing powers of fast computers. A basic cognitive radar (BCR) contains a global feedback link encompassing the receiver, the transmitter, and the environment along with the signal processing blocks in the transmitter and the receiver. Moreover, unlike BCR, cognitive radar has memory blocks in it [4]. Figure 1 shows the basic cognitive cycle.

The basic cognitive cycle begins with the transmitter illuminating the radar environment. Radar returns contain the information about the targets/interceptors in the space. The receiver senses the radar environment by continuously interacting with it to get the necessary information regarding targets/interceptors. This information is sent as a feedback from the receiver to the transmitter. The transmitter of 


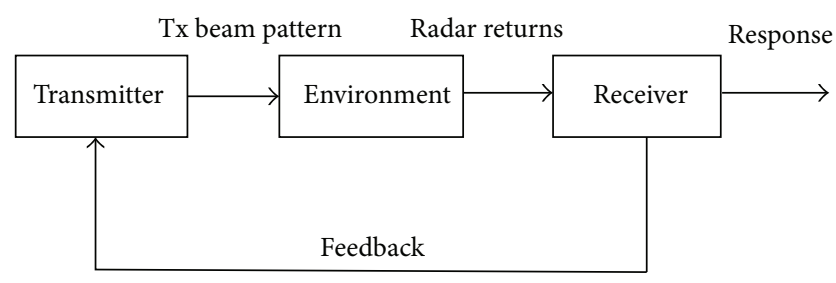

FIgURE 1: Basic cognitive cycle.

cognitive radar contains the intelligent signal processing block which allows changing its properties for transmitting an appropriate beam pattern in accordance with the statistical variations in the environment. This cycle keeps on repeating $[3,4]$.

The active transmitting antenna may contain an array of transmitting elements. The transmitting array elements have the ability to form a beam pattern that can concentrate a high amount of energy in the desired direction, while putting nulls in the undesired direction to cancel jammers or unwanted signals [5]. These array antennas also have the ability to steer the beam towards any desired direction in space [6]. The high gain of this active array antenna helps the radar system in better detection of targets but, at the same time, is very likely to be detected by interceptors. Hence, it is very common that interceptors may degrade or destroy the capabilities of these radars [7]. This competition between the radar systems and their opposing forces is termed as electronic warfare (EW) [8]. Numerous waveform design techniques, for attaining LPI property, which have been employed to encounter this challenge, are given in the literature. Mainly, three techniques are used to achieve LPI property and securing the radar from being detected by interceptors. These techniques include using (i) high duty cycle waveforms for spreading energy in temporal domain, (ii) wide bandwidth waveforms for spreading energy in frequency domain, and (iii) broader transmit beam patterns for spreading energy in spatial domain. More often the combination of these techniques is much helpful also for ensuring LPI property [7, 9-12]. In [7], a novel transmit array beamforming approach with LPI property has been introduced employing phased array antennas.

In this paper, a novel design of the hybrid cognitive phased array radar with transmit beamforming having LPI property is presented. The receiver array of the proposed radar gets the radar returns to estimate the interceptor range and the DOA using EKF and GA based evolutionary computing technique, respectively. This target/interceptor range and the direction are sent as a feedback to the transmitter. The transmitter of the proposed radar contains a memory block and a selector. At the transmitter, the traditional high gain scanned beam patterns are spoiled to get a set of low gain basis patterns. The reduced peak power of these basis patterns reduces the radar visibility. These basis patterns are thus combined using complex weights to synthesize the original high gain beam pattern for unaffected array detection performance. The GA block calculates these complex weights offline, for three different numbers of array elements with various possible directions, and are kept in the transmitter

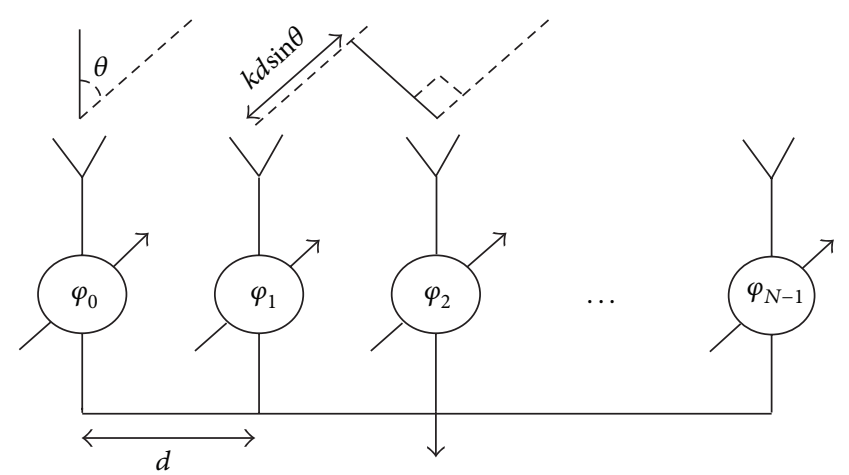

FIGURE 2: Uniform linear array having $N$ elements, precalculated set of $\varphi_{n}$ to spoil beams, interelement distance $d$, wave number $k$, and target direction $\theta$.

memory block. The higher the range of the target, the higher the number of array elements to get high directional gain. The selector block of the transmitter examines the range information and proposes an appropriate number of antenna elements using preset lookup table. The spoiling phase-shift block gives the appropriate phase shifts to get the spoiled basis beam patterns. Then the selector interacts with the corresponding subblock of memory for getting an appropriate weight vector to generate a high gain beam pattern using these set of basis patterns.

The paper is organized as follows. Section 2 provides the details on algorithms of the literature (i.e., LPI array beamforming and genetic algorithm steps). Section 3 describes system design, Section 4 is for simulations and results, and Section 5 concludes the paper.

\section{Algorithms of Literature}

2.1. Array Beamforming with LPI Property. In [7], Lawrence presented a novel approach for transmit array beamforming that offers LPI for surveillance radar systems using phased array antennas. In this approach the high gain scanned antenna beam patterns throughout the search region are spoiled to get series of low-gain spoiled basis patterns by feeding in an extra calculated phase shift to each array element. This low-gain basis pattern reduces the radar's probability of being detected by opposing forces. To get unaffected detection performance than that of high gain scanned array, these spoiled beam patterns are coherently combined using complex weights to direct the concentrated energy towards the target/interceptor direction. Figure 2 shows a uniform linear array (ULA) with $N$ array elements. The interelement distance is taken as $d$ whereas the calculated values of $\varphi_{n}$ phase shifts are applied to spoil the high gain beam patterns.

The detection performance of this approach remains unchanged due to the fact that the total energy incident on the target is same as before. The pattern of an $N$ elements ULA having interelement distance taken as $d=\lambda / 2$ is written as

$$
h\left(\psi_{0}\right)=\sum_{n=0}^{N-1} e^{j n \psi_{0}}
$$


where $k=2 \pi / \lambda$ and $\psi_{0}=k d \sin \theta-k d \sin \theta_{0}$. This transmit beam pattern has a high gain lobe in the direction of the spatial angle $\theta_{0}$ measured from the broadside of the array. This high gain beam pattern can be scanned throughout the search region $[-\pi / 2, \pi / 2]$ by applying a linear progressive phase shift across the array whereas fundamental phase scan shift is taken as $\gamma=2 \pi / N$. For steering of beam pattern in the search region, we define $\psi_{m}=\psi_{0}+m \gamma, m=0,1,2,3, \ldots,(N-$ 1). The fundamental beam pattern is shown in (1), while the remaining set of $N-1$ scanned pattern of the fundamental beam pattern throughout the search region can be expressed as

$$
h\left(\psi_{m}\right)=\sum_{n=0}^{N-1} e^{j n \psi_{m}} ; \quad m=1,2,3, \ldots,(N-1) .
$$

These high gain scanned patterns are spoiled in a way that their peak power in any direction reduces significantly to ensure the LPI property. The quadratic phase variance technique is employed to find such a set of phase shifter values $\left(\varphi_{n}\right)$ that reduces peak power of the high gain beam pattern. The set of $N$ spoiled/basis beam pattern $l\left(\psi_{m}\right), m=$ $0,1,2,3, \ldots,(N-1)$, is expressed as

$$
\begin{gathered}
l\left(\psi_{0}\right)=1+e^{j \varphi_{1}} e^{j \psi_{0}}+e^{j \varphi_{2}} e^{j 2 \psi_{0}}+e^{j \varphi_{3}} e^{j 3 \psi_{0}} \\
+\cdots+e^{j \varphi_{N-1}} e^{j(N-1) \psi_{0}} \\
l\left(\psi_{1}\right)=1+e^{j \varphi_{1}} e^{j \psi_{1}}+e^{j \varphi_{2}} e^{j 2 \psi_{1}}+e^{j \varphi_{3}} e^{j 3 \psi_{1}} \\
+\cdots+e^{j \varphi_{N-1}} e^{j(N-1) \psi_{1}} \\
\vdots \\
l\left(\psi_{N-1}\right)=1+e^{j \varphi_{1}} e^{j \psi_{N-1}}+e^{j \varphi_{2}} e^{j 2 \psi_{N-1}}+e^{j \varphi_{3}} e^{j 3 \psi_{N-1}} \\
+\cdots+e^{j \varphi_{N-1}} e^{j(N-1) \psi_{N-1}} .
\end{gathered}
$$

These $N$ steered versions of the fundamental beam patterns are linearly independent [7]. As it is desired that the performance of radar should remain unchanged, while ensuring the LPI property at the same time, so the high gain beam pattern towards the desired direction is synthesized by linearly combining these spoiled beams $l\left(\psi_{m}\right)$ using appropriate weights:

$$
\begin{aligned}
& {\left[\begin{array}{c}
\widehat{h}\left(\psi_{0}\right) \\
\widehat{h}\left(\psi_{1}\right) \\
\cdot \\
\cdot \\
\cdot \\
\hat{h}\left(\psi_{N-1}\right)
\end{array}\right]} \\
& =\left[\begin{array}{cccccc}
w_{0,0} & w_{0,1} & \cdot & \cdot & \cdot & w_{0, N-1} \\
w_{1,0} & w_{1,1} & & & & w_{1, N-1} \\
\cdot & \cdot & \cdot & & \cdot \\
\cdot & \cdot & & \cdot & & \cdot \\
\cdot & \cdot & & \cdot & \cdot \\
w_{N-1,0} & w_{N-1,1} & \cdot & \cdot & \cdot & w_{N-1, N-1}
\end{array}\right]\left[\begin{array}{c}
l\left(\psi_{0}\right) \\
l\left(\psi_{1}\right) \\
\cdot \\
\cdot \\
\cdot \\
l\left(\psi_{N-1}\right)
\end{array}\right]
\end{aligned}
$$

2.2. Genetic Algorithm Based Estimation Algorithm. There are a lot of engineering problems that are very complex in nature and are not easy to solve using conventional and analytical techniques. Evolutionary computation techniques give the solution to that type of problems. Researchers show their keen interest regarding their applications towards the fields of biomedical medical engineering, radars and sonars, and so forth [13]. The heuristic techniques have a vital advantage of avoiding the local minima along with the best performance in low signal to noise scenarios [14]. Differential evolution (DE), genetic algorithm (GA), particle swarm optimization (PSO), and so forth are examples of these techniques. Often hybridization of these techniques with local optimizers like pattern search (PS) performs better in some scenarios [15]. The GA computation technique uses natural selection and genetics mechanism to find the optimized solution [13]. GA initializes a random solution set, called a population. Each individual in generated population is called a chromosome. The fitness function defines the relationship between desired and estimated solutions to the different types of problems. The general steps of the GA are given in the literature [13-15].

\section{System Design}

The block diagram of the proposed radar contains different subblocks in the transmitter and the receiver ends. The transmit beam illuminates the radar environment. The radar environment has different types of targets/interceptors. The radar returns from the environment come at the receiver. The receiver has the EKF algorithm block and the GA based evolutionary computing block for estimating the range of target and the DOA, respectively. This estimated range $(\widehat{R})$ and the DOA $\left(\widehat{\theta}_{0}\right)$ information are sent back as an information feedback to the transmitter. Figure 3 shows the block diagram of the proposed radar.

The feedback is easy to implement as it is assumed that the receiver and the transmitter of the proposed radar are collocated. The transmitter end of the proposed radar contains subblocks. The phased array antenna in the transmitter block, with a predetermined extra phase shifter value for each element, has been designed to get spoiled/low gain basis pattern to ensure the LPI property. In order to maintain its detection performance, a suitable set of complex weights is needed to linearly combine this spoiled pattern to synthesized high gain beam pattern towards the desired direction. The GA based evolutionary computing block computes the complex weights, offline. Offline calculated weights, for three different numbers of array elements, that is, $N=24,32,40$, are stored in the corresponding memory block of the transmitter. The maximum value of gain to the desired direction is equal to the number of array antenna elements. Hence, for better detection performance, the higher the range, the higher the gain requirement to detect a target precisely. Selector examines the estimated range and selects the suitable number of array elements using a predetermined lookup table. Once the number of elements has been determined, selector interacts with phase shifter values block to set corresponding phase shifts to spoil beam and simultaneously interacts with 


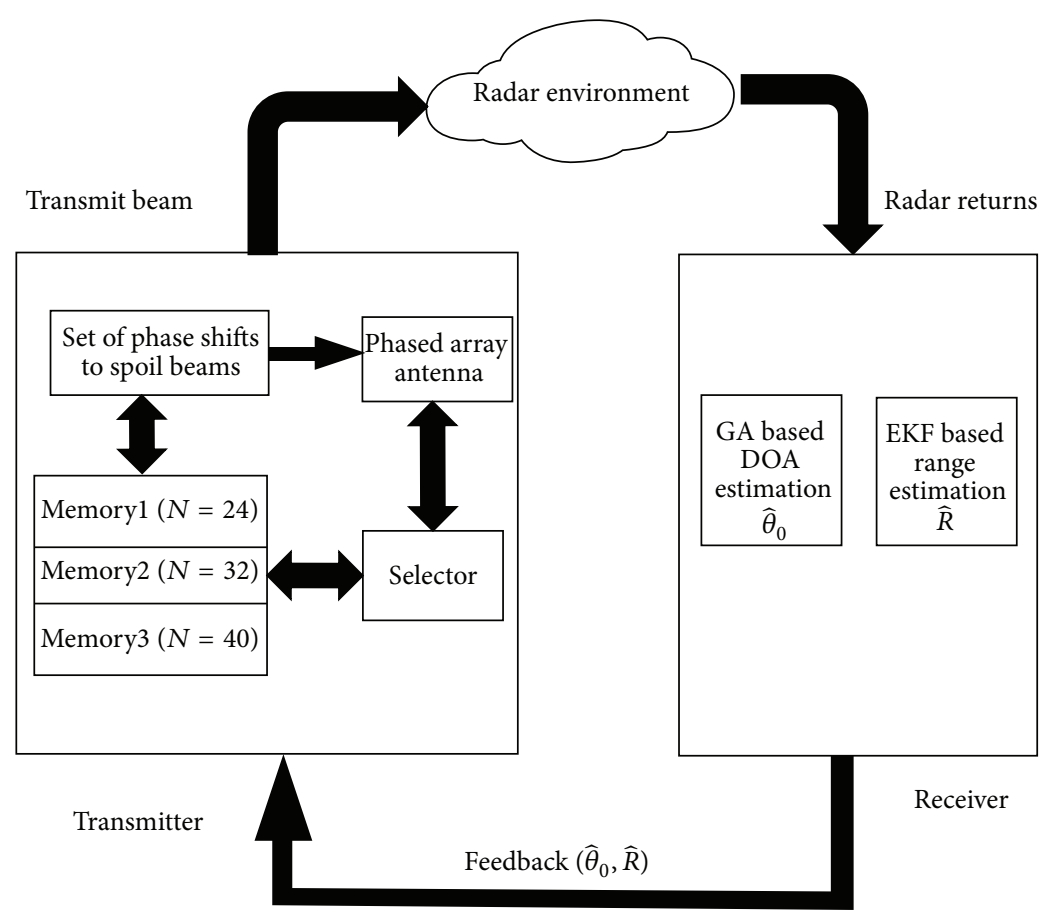

Figure 3: Block diagram of the proposed radar.

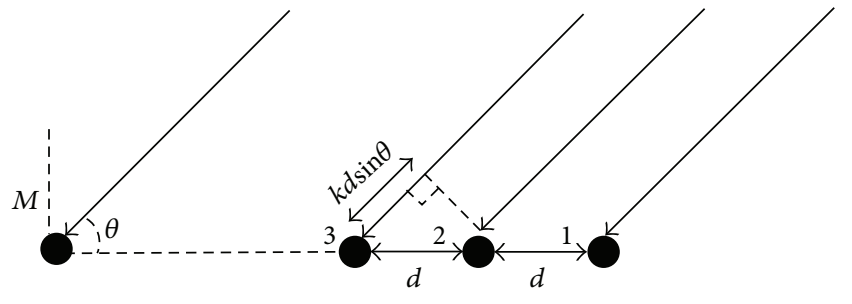

FIGURE 4: A uniform linear receiving array with $M$ elements.

the memory, to get the appropriate weights for synthesizing high gain beam pattern using low gains basis patterns in the desired direction.

\subsection{Receiver End}

3.1.1. Direction of Arrival Estimation. In the current era, direction of arrival (DOA) estimation is one of the important research concerns in the field of signal processing and antenna arrays. In [15], for joint estimation of DOA, range, and amplitude, DE and PSO are hybridized with PS for far field targets using uniform linear array (ULA). The receiver end uses the GA based computing technique for estimating the DOA for far field target. Fitness function defined as the mean square error between the desired and estimated direction of arrival using ULA. Figure 4 shows the receiving ULA having $M$ elements.

The interelement distance is taken as $d$. It is assumed that $L$ far field sources impinge on ULA from various elevation angles, respectively. The output at $m$ th element of the array, provided $L \leq M$, is given as

$$
f_{m}=\sum_{l=1}^{L} A_{l} e^{\left(-j k d(m-1) \sin \left(\theta_{l}\right)\right)}+n_{m}
$$

where $m=1,2, \ldots, M ; n_{m}$ is an additive white Gaussian noise added to $m$ th element and $A_{l}$ is the amplitude of $l$ th source. The sources are considered to be narrowband with frequency $w_{0}$. The estimated output at the $m$ th element is given as

$$
\widehat{f}_{m}=\sum_{l=1}^{L} \widehat{A}_{l} e^{\left(-j k d(m-1) \sin \left(\widehat{\theta}_{L+1}\right)\right)} .
$$

General GA steps are used to find the DOA. The fitness function defines the mean square error $E_{m}$ between desired and estimated response and is given as

$$
E_{m}=\frac{1}{M} \sum_{m=1}^{M}\left|f_{m}-\widehat{f}_{m}\right|^{2} \quad \text { where } m=1,2, \ldots, M \text {. }
$$

3.1.2. Range Estimation Using EKF. EKF is used for nonlinear dynamic model parameter estimation. The complete twostage process, that is, linearization followed by Kalman filter (KF), is known as EKF [16-19]. Observed data at any discrete time $k$ is known as observation $z_{k}$, while the output of any system that completely describes the behavior of that system is known as state $\mathbf{x}_{k}$ [18]. The observation vector $\mathbf{z}_{k}$, where $\mathbf{z}_{k}=\left[z_{1}, z_{2}, \ldots, z_{k}\right]$, is used to estimate the state. EKF estimates the output in two steps, it predicts the state using $\mathbf{z}_{k-1}$ observation vector and updates this state to new state 
based on new observation $z_{k}$ [19]. The conditional mean of the state $\mathbf{x}_{k}$ is denoted as

$$
\widehat{\mathbf{x}}_{k \mid k}=E\left[\mathbf{x}_{k} \mid \mathbf{z}_{k}\right] .
$$

$\left\{x_{k}\right\}$ and $\left\{z_{k}\right\}$ are assumed zero mean and jointly Gaussian stochastic processes, that is, $E\left[x_{k}\right]=E\left[z_{k}\right]=0, \forall k$; hence the conditional estimate $\widehat{\mathbf{x}}_{k \mid k}$ is orthogonal projection of $\mathbf{x}_{k}$ on the space spanned by these observations $z_{1}, z_{2}, \ldots, z_{k}[20-$ 24]. The conditional covariance matrix of the state vector is denoted as

$$
\widehat{\mathbf{P}}_{k \mid k}=E\left[\left(\mathbf{x}_{k}-\widehat{\mathbf{x}}_{k \mid k}\right)\left(\mathbf{x}_{k}-\widehat{\mathbf{x}}_{k \mid k}\right)^{\prime} \mid \mathbf{z}_{k}\right] .
$$

The process equation of a nonlinear dynamic model is given as

$$
\mathbf{x}_{k+1}=\mathbf{f}\left(k, \mathbf{x}_{k}\right)+\mathbf{w}_{k} .
$$

$\mathbf{f}\left(k, \mathbf{x}_{k}\right)$ denotes the nonlinear transition matrix which takes the state $\mathbf{x}_{k}$ from time $k$ to $k+1$. $\mathbf{w}_{k}$ is process noise and is assumed to be zero mean Gaussian noise with covariance matrix as

$$
\mathbf{E}\left[\mathbf{w}_{k} \mathbf{w}_{n}^{T}\right]=\mathbf{Q}_{k} \delta_{n k} .
$$

The measurement equation is given as

$$
\mathbf{z}_{k}=\mathbf{h}\left(k, \mathbf{x}_{k}\right)+\mathbf{v}_{k} .
$$

$\mathbf{h}\left(k, \mathbf{x}_{k}\right)$ denotes measurement matrix for nonlinear system and $\mathbf{v}_{k}$ is measurement noise assumed to be zero mean Gaussian noise with covariance matrix given as

$$
\mathbf{E}\left[\mathbf{v}_{k} \mathbf{v}_{n}^{T}\right]=\mathbf{R}_{k} \delta_{n k} .
$$

As EKF linearizes the nonlinear model and then applies KF, the linearization is obtained by constructing the following two matrices at first stage:

$$
\mathbf{F}_{k+1, k}=\left.\frac{\partial \mathbf{f}\left(k, \mathbf{x}_{k}\right)}{\partial \mathbf{x}}\right|_{\mathbf{x}=\widehat{\mathbf{x}}_{k}}, \quad \mathbf{H}_{k}=\left.\frac{\partial \mathbf{h}\left(k, \mathbf{x}_{k}\right)}{\partial \mathbf{x}}\right|_{\mathbf{x}=\widehat{\mathbf{x}}_{k-1}} .
$$

After that, these calculated matrices are used in first-order Taylor series approximation of nonlinear functions $\mathbf{F}\left(k, \mathbf{x}_{k}\right)$ and $\mathbf{H}\left(k, \mathbf{x}_{k}\right)$ around $\mathbf{x}_{k}$ and $\mathbf{x}_{k-1}$, respectively [22]:

$$
\begin{gathered}
\mathbf{F}\left(k, \mathbf{x}_{k}\right) \approx \mathbf{F}\left(\mathbf{x}, \widehat{\mathbf{x}}_{k}\right)+\mathbf{F}_{k+1, k}\left(\mathbf{x}, \widehat{\mathbf{x}}_{k}\right), \\
\mathbf{H}\left(k, \mathbf{x}_{k}\right) \approx \mathbf{H}\left(\mathbf{x}, \widehat{\mathbf{x}}_{k-1}\right)+\mathbf{H}_{k+1, k}\left(\mathbf{x}, \widehat{\mathbf{x}}_{k-1}\right) .
\end{gathered}
$$

After that, KF is applied to get the estimate for future states. Kalman prediction equation is given as

$$
\begin{gathered}
\widehat{\mathbf{x}}_{k \mid k-1}=\mathbf{F}\left(k, \mathbf{x}_{k}\right) \widehat{\mathbf{x}}_{k-1 \mid k-1}, \\
\widehat{\mathbf{P}}_{k \mid k-1}=\mathbf{F}\left(k, \mathbf{x}_{k}\right) \widehat{\mathbf{P}}_{k-1 \mid k-1} \mathbf{F}\left(k, \mathbf{x}_{k}\right)^{T}+\mathbf{Q}_{k-1} .
\end{gathered}
$$

The update equation is given as

$$
\begin{gathered}
\widehat{\mathbf{x}}_{k \mid k}=\widehat{\mathbf{x}}_{k \mid k-1}+\mathbf{G}_{k}\left(\mathbf{z}_{k}-\mathbf{H}\left(k, \mathbf{x}_{k}\right) \widehat{\mathbf{x}}_{k \mid k-1}\right), \\
\widehat{\mathbf{P}}_{k \mid k}=\widehat{\mathbf{P}}_{k \mid k-1}-\mathbf{G}_{k}\left(\mathbf{H}\left(k, \mathbf{x}_{k}\right) \widehat{\mathbf{P}}_{k \mid k-1}\right),
\end{gathered}
$$

where $\mathbf{G}_{k}$ is the Kalman Gain and is given as

$$
\mathbf{G}_{k}=\widehat{\mathbf{P}}_{k \mid k-1} \mathbf{H}\left(k, \mathbf{x}_{k}\right)^{T}\left(\mathbf{H}\left(k, \mathbf{x}_{k}\right) \widehat{\mathbf{P}}_{k \mid k-1} \mathbf{H}\left(k, \mathbf{x}_{k}\right)^{T}+\mathbf{R}_{k}\right)^{-1} .
$$

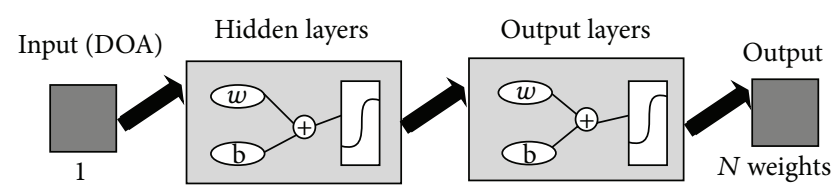

FIGURE 5: Neural Network block diagram (memory).

3.2. Transmitter End. The transmitter of the proposed cognitive radar changes its attributes/properties in accordance with the feedback sent from the transmitter. The transmitter end contains four subblocks which are the phased array antenna, the phase shifter values block (to spoil beam), the memory block, and the selector.

3.2.1. GA Based Calculation of Complex Weights (Offline). GA is a promising candidate of evolutionary computing techniques. The need is to compute the set of complex weights using the genetic algorithm (GA). The set of spoiled beams $l\left(\psi_{m}\right)$ is represented in vector form as $\mathbf{l}_{N \times 1}$. It is desired to generate the high gain beams $h\left(\psi_{m}\right)$ using the linear combination of these spoiled beams. The vector $h\left(\psi_{m}\right)$ and complex weights matrix is denoted by $\widehat{\mathbf{h}}_{N \times 1}$ and $\mathbf{W}_{N \times N}$, respectively. Equation (4) can be expressed as

$$
\widehat{\mathbf{h}}=\mathbf{W l} \text {. }
$$

Different from the author in [7], GA is used for finding the set of complex weights for synthesizing the high gain beam pattern utilizing the basis beam patterns. This procedure will give the complex weights which will be stored in the memory. The fitness function that defines the mean square error $D_{m}$ between the desired and the estimated response is given as

$$
D_{m}=\frac{1}{M} * \sum_{i=1}^{M}\left|h_{m}(i)-\widehat{h}_{m}(i)\right|^{2} ; \quad m=0,1,2, \ldots,(N-1),
$$

where $\widehat{\mathbf{h}}_{m}=\sum_{j=0}^{N-1} \mathbf{W}_{m, j} l\left(\psi_{j}\right) ; \widehat{\mathbf{h}}_{m}$ denotes $m$ th element of the vector $\widehat{\mathbf{h}} ; M=$ Length $\left(\widehat{\mathbf{h}}_{m}\right)$. These weights are calculated for three different numbers of array elements and stored in the memory for future use.

3.2.2. Memory. The memory within the transmitter side called executive memory. This memory sees the overall radar environment indirectly through the feedback information received [4]. The memory used in the proposed transmitter end only contains the sets of appropriate weights for generating the high gain beam patterns using the low gain basis patterns while ensuring the LPI property. A neural network is the simplest choice to design and implement this type of memory [25]. Back propagation algorithm is used to train this neural network [26].

Once the estimated DOA and the estimated range are known to the transmitter end, the task is to get the appropriate weights for synthesizing a high gain beam pattern. Figure 5 shows the chosen neural network that takes this DOA as single value input and gives out the corresponding $N$ weights. 
3.2.3. Selector. The selector block in the transmitter takes input of the estimated range and the estimated DOA. It selects the appropriate number of array elements using a predefined lookup table for obtaining a specific gain. Simultaneously, it gives this input to the phase shifter values block to get the required number of spoiling phase shifter values. After that it gets the appropriate weights from memory to synthesize an appropriate beam pattern that holds the LPI property in it.

\section{Simulations and Results}

This section discusses the simulation and the results of the presented novel radar design. The simulation results of each block in the receiver and the transmitter have been shown, respectively. MATLAB 2012 has been used for simulations while nntool and optimtool, MATLAB toolboxes, are used for implementing memory and the GA based simulations, respectively.

4.1. Interceptor/Target Range Estimation. EKF is used for estimation of the target range $(\widehat{R})$ for a nonlinear track. For simulation purpose $\mathbf{x}_{k}$ state defines the Euclidian distance of the target from a fixed point that is taken as the range of target $(\widehat{R})$. Figure $6(\mathrm{a})$ shows the noisy observations for estimation of the state at the discrete time intervals $(k)$. These observations are obtained by adding the AWGN to the original track values maintaining a constant signal to noise ratio (SNR). Figure 6(b) shows the performance of EKF for tracking this nonlinear target track. Increasing the SNR significantly decreases the root mean square error (RMSE) value between the original and the estimated range values. Figure 6(c) shows the performance comparison of the EKF and the Kalman filter (KF) for tracking a nonlinear track.

EKF estimates the original track trajectory using these noisy observations shown in Figure 6(a). This comparison result in Figure 6(c) gives a clear picture that EKF estimates the nonlinear track range efficiently and thus validating the choice of the EKF for the proposed radar.

4.2. DOA Estimation at Receiver. Simultaneously, the proposed radar receiver takes the radar returns as input to the DOA estimation block. The MATLAB optimtool toolbox is used for finding the optimum solution based on GA. Table 1 gives the parameter settings of the GA for estimating the DOA of the target/interceptor.

For the sake of simulation, it is assumed that there is only one source in the far field. Table 2 shows the accuracy analysis of DOA estimation of that target which is estimated using GA. The DOA angle values are taken in degrees. To validate the results, the GA performance at five different discrete sessions is evaluated.

4.3. Feedback. The feedback link acts as a tunnel to send the target position (the estimated range and the estimated DOA) from the receiver to the transmitter. The proposed cognitive radar transmitter acts according to the feedback and wants to focus the concentrated energy towards the target's direction simultaneously enjoying the advantages of LPI. The number
TABLE 1: Parameter settings of GA.

\begin{tabular}{lc}
\hline & Genetic algorithm \\
Parameters & Settings \\
\hline Population size & 20 \\
Chromosome size & 1 \\
Creation function & Uniform \\
Selection function & Stochastic uniform \\
Reproduction crossover fraction & 0.6 \\
Mutation function & Adaptive feasible \\
Crossover function & Heuristic \\
Migration direction & Both \\
Hybrid function & None \\
Number of generations & 500 \\
Function tolerance & $1 e-22$ \\
Nonlinear constraint tolerance & $1 e-22$ \\
\hline
\end{tabular}

TABLE 2: Accuracy analysis of the target direction estimation.

\begin{tabular}{lccccc}
\hline \multicolumn{5}{c}{ Estimation of DOA using GA (angles in degrees) } \\
\hline Desired DOA & $0^{\circ}$ & $45^{\circ}$ & $75^{\circ}$ & $-30^{\circ}$ & $-45^{\circ}$ \\
Estimated DOA & $0.0021^{\circ}$ & $45.0812^{\circ}$ & $75.0482^{\circ}$ & -30.1012 & -45.0816 \\
\hline
\end{tabular}

of elements to form an array is chosen by keeping in view the estimated range of the target.

4.4. LPI Array Beamforming. A 32-element $(N=32)$ uniform linear array with interelement distance $d=\lambda / 2$ is taken. Figure 7 (a) shows the gain comparison between fundamental high gain beam pattern and spoiled/basis beam pattern. This fundamental high gain beam pattern is spoiled using a predefined set of phase shifter values $\varphi_{n}$ applied to ULA. The set of phase shifter values $\varphi_{n}$ is calculated using a quadratic phase variance technique where the constraint is to minimize the gain [7]. This calculated set applies to each of the scanned patterns to get the low gain basis patterns. Figure 7(a) shows that the high gain beam pattern has a gain of $15 \mathrm{dBi}$ approximately while the spoiled version of this high gain beam has $1.7 \mathrm{dBi}$ approximately. gain value that is significantly lower than that of ULA. Figure 7(b) shows the scanned patterns of this fundamental high gain pattern over all the surveillance region. Figure 7 (c) shows the corresponding scanned spoiled beam patterns. Figure $7(d)$ shows the high gain beam synthesis performance of the GA and the method used in [7].

Figure $7(\mathrm{~d})$ gives a clear picture that the GA method used for synthesis of high gain shows almost equal performance than that method used in [7]. The advantage of the GA is the low complexity specifically by avoiding the matrix inversions.

4.5. GA Based Weight Calculation (Offline). Once the set of spoiled beam patterns is generated, the need is to find an appropriate set of weights that can synthesize the high gain beam patterns in the desired directions. MATLAB optimtool toolbox with GA is used for finding the optimum weights. Table 3 shows the parameter settings of the GA for $N=32$. 


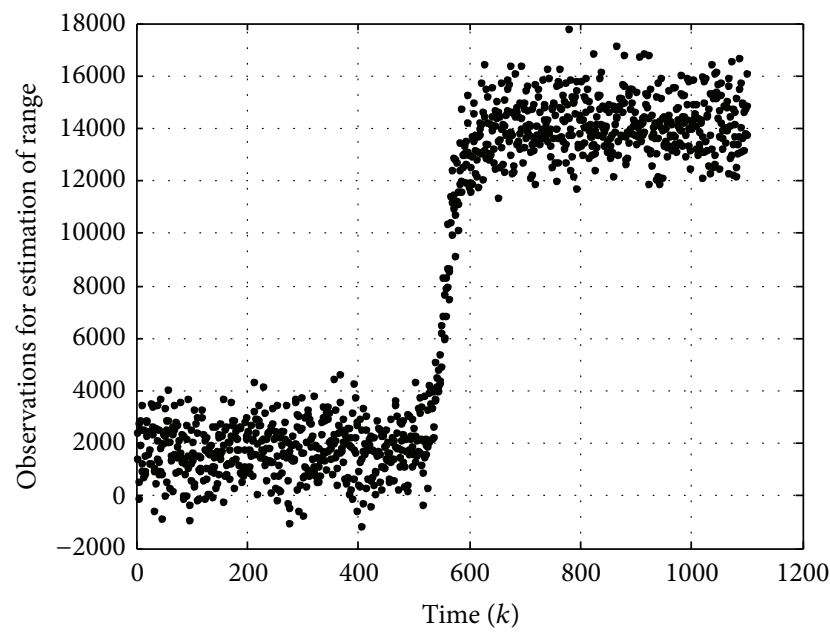

(a)

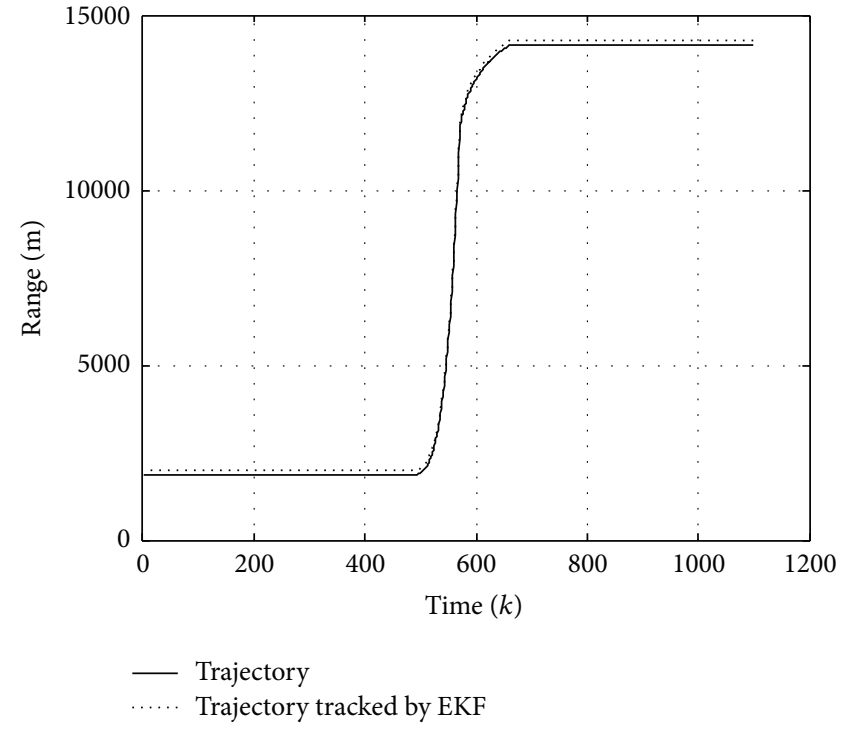

(b)

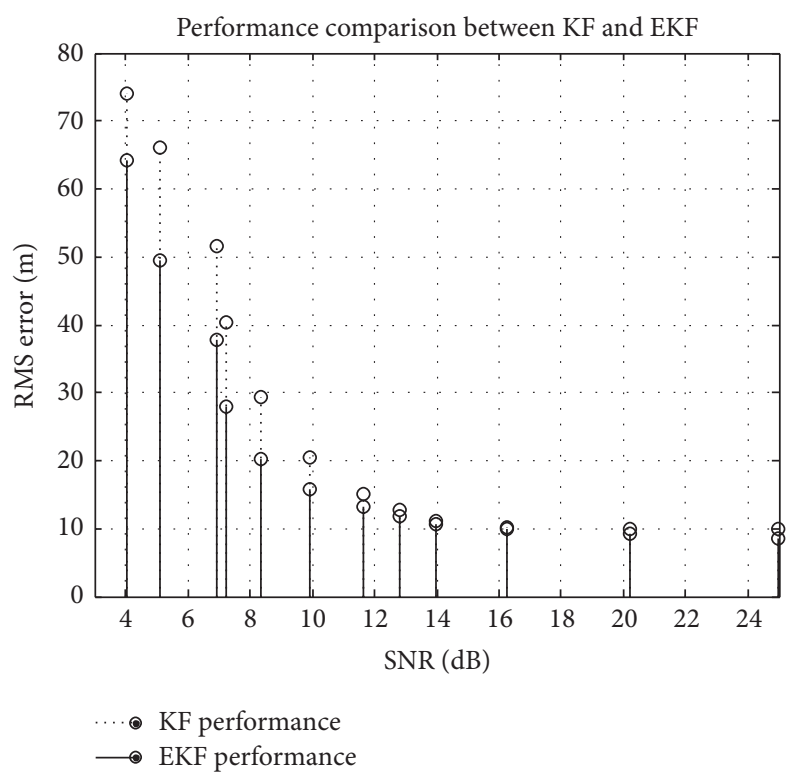

(c)

FIGURE 6: (a) Observations; (b) the target range estimation using EKF; (c) SNR versus RMS Error.

The GA finds the best weights for three fixed sizes of the array $(N=24,32,40)$ for synthesizing the high gain beam pattern using low gain basis patterns. These weights are stored in the memory for future use.

4.6. Selector. These stored weights in the memory are used time by time as per requirement. When the range and the DOA are received as feedback, selector examines the range to set the appropriate number of array elements using a lookup table. Table 4 shows the lookup table maintained at the transmitter.

The phase shifter values block in transmitter gives corresponding $N$ phase shift values to array antenna for spoiling the beam patterns. After that, selector interacts with the concerning block of memory to get the appropriate set of weights to allow the phased array antenna to synthesize a high gain beam pattern towards the target direction.

4.7. Beamforming (Online). In uniform linear array beamforming, maximum gain value (directivity) towards $\theta_{0}$ (target direction) is given by

$$
G\left(\theta_{0}\right)_{\max }=\left|\mathbf{a}^{\mathbf{H}}\left(\theta_{0}\right) \mathbf{a}(\theta)\right|=N,
$$

where $\mathbf{a}(\theta)$ is transmit steering vector, while $\mathbf{a}^{\mathbf{H}}\left(\theta_{0}\right)$ is the target steering vector. It is clear from the equation that the increase in the array element leads to the increased directed 


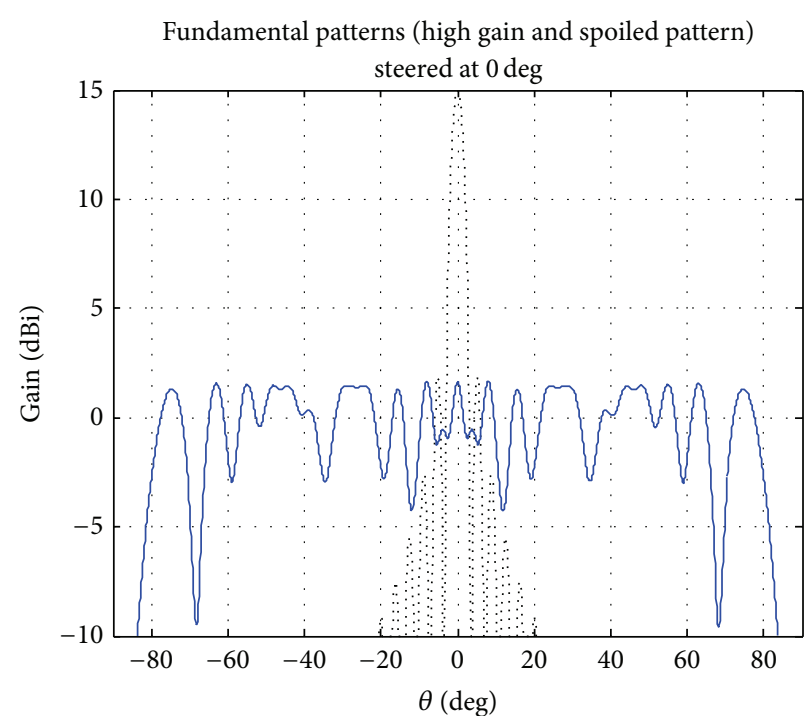

..... Fundamental ULA pattern

- Spoiled basis pattern

(a)

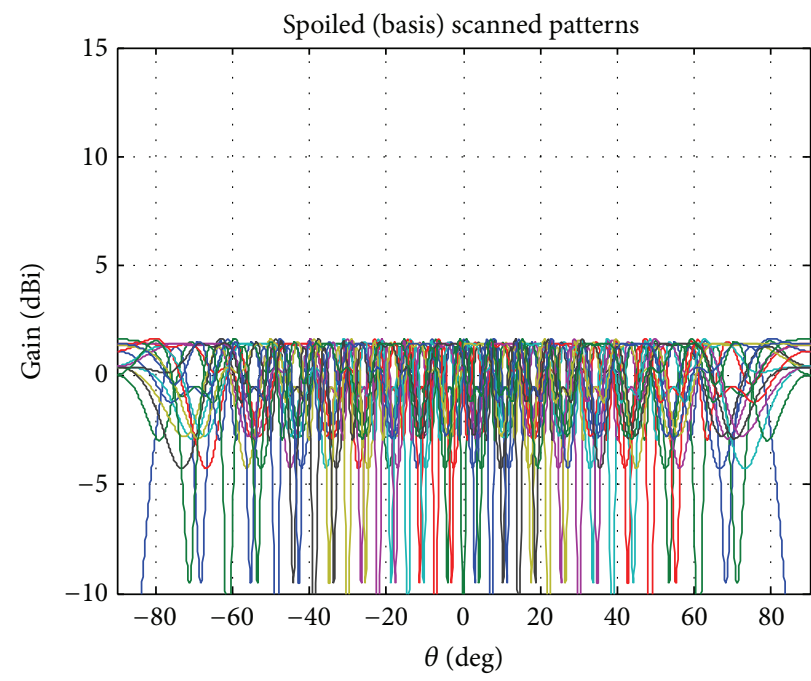

(c)

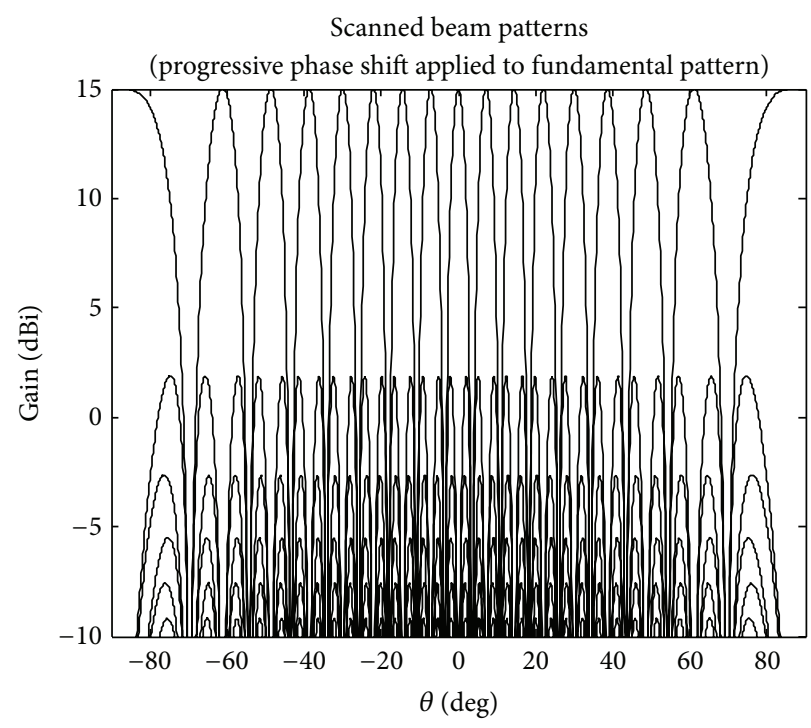

(b)

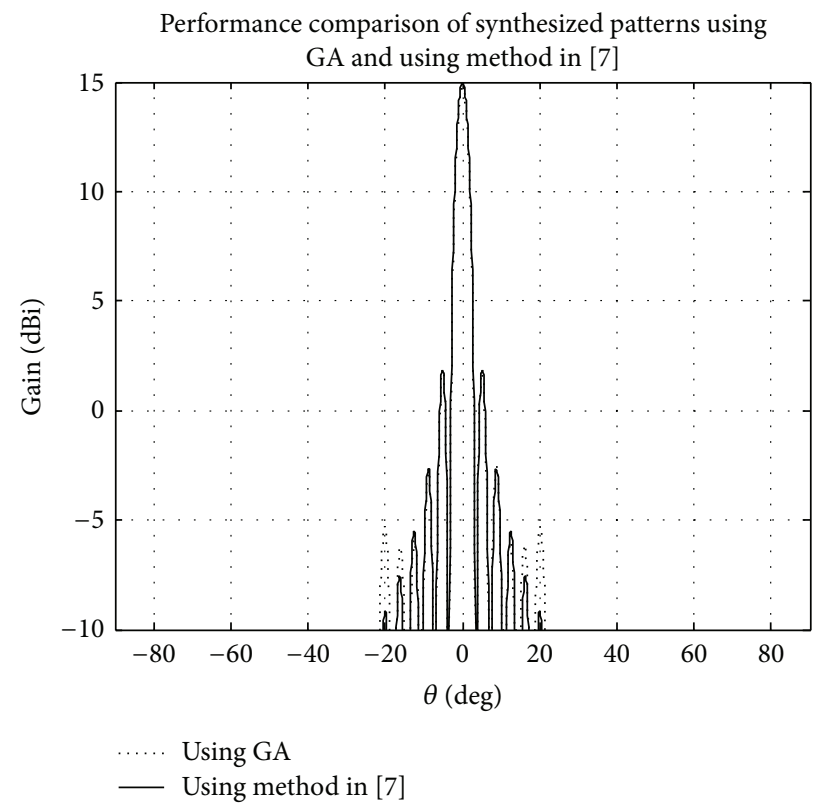

(d)

FIGURE 7: (a) Gain comparison between fundamental pattern and fundamental spoiled pattern; (b) scanned high gain beam patterns; (c) all spoiled beam patterns; (d) pattern synthesis performance of the GA and the author's method in [7].

beam towards the target. So, if the range of the target is high, a high number of array elements are required for directing the sharper beam towards it for better detection performance. Similarly, the low range requires less number of array elements to generate the directed beam towards that target.

At first, for simulation purposes it is shown that the target range is constant (i.e., $\widehat{R}=8000 \mathrm{~m}$ ) and the DOAs at different discrete times are $0^{\circ}, 45^{\circ}$, and $-45^{\circ}$, respectively. Figure 8 shows the synthesized beam using GA towards the different estimated DOAs.
After that, for simulation purposes, the transmitter of the proposed radar gets the information about the target at different estimated ranges and different estimated directions. The selector observes the range limit and decides the number of array elements for individual position of the target and interacts with the concerned memory to get the appropriate weights to generate the high gain beam patterns towards desired directions. Figure 9 shows the synthesized high gain beam patterns for different ranges (i.e., $4 \mathrm{~km}, 7 \mathrm{~km}$, and $12 \mathrm{~km})$ and directions of the target $\left(0^{\circ}, 30^{\circ}\right.$, and $\left.60^{\circ}\right)$ at a different discrete time, respectively. It is clear from Figure 9 
TABLE 3: Parameter settings of the GA.

\begin{tabular}{lc}
\hline & Genetic algorithm \\
Parameters & Settings \\
\hline Population size & 40 \\
Chromosome size & 64 \\
Creation function & Uniform \\
Selection function & Stochastic uniform \\
Reproduction crossover fraction & 0.6 \\
Mutation function & Adaptive feasible \\
Crossover function & Heuristic \\
Migration direction & Both \\
Hybrid function & None \\
Number of generations & 1000 \\
Function tolerance & $1 e-10$ \\
Nonlinear constraint tolerance & $1 e-10$ \\
\hline
\end{tabular}

TABLE 4: Lookup table maintained by the selector.

Lookup table (range limits versus number of array elements) Range (meters) $\quad 0-5000 \quad 5000-10000 \quad 10000-15000$

$N$

24

32

40

that when the range of the target is high, a more directed beam is generated by using greater number of array elements.

Figure 10 shows the $d B i$ gain of the respective beam patterns shown in Figure 9. The high directed gain value is generated by ULA in the direction of the high range target.

Increase in the target range decreases the incident power on the target. The proposed cognitive PAR changes the number of elements according to the range value of target to maintain a reasonable incident power (directivity) for better detection performance. Figure 11 shows the comparison of the incident power on the target between the proposed radar and the fixed elements uniform linear array.

On the other hand, when the range of the target is low, the proposed radar uses a lesser number of elements $(N)$ that results in the reduced length of the transmitter and target steering vectors. Hence, the computational complexity is reduced than that of the fixed sized transmitter array.

Another major advantage of the proposed array radar is that it avoids the continuous scanning of the surveillance region; rather it only generates the beam pattern in accordance with the target position sent as feedback from the receiver. Hence, the computational complexity reduces even more.

\section{Conclusion}

In this paper, a novel hybrid cognitive phased array radar design having LPI transmit array beamforming is presented. The high gain property of PAR, having the advantage of efficient detection of the target/interceptor, reveals its position to the interceptor. Hence to hide a radar from interceptors, a known LPI technique in the literature is applied at the transmitter. PAR technology having LPI is hybridized with the emerging cognitive radar technology.
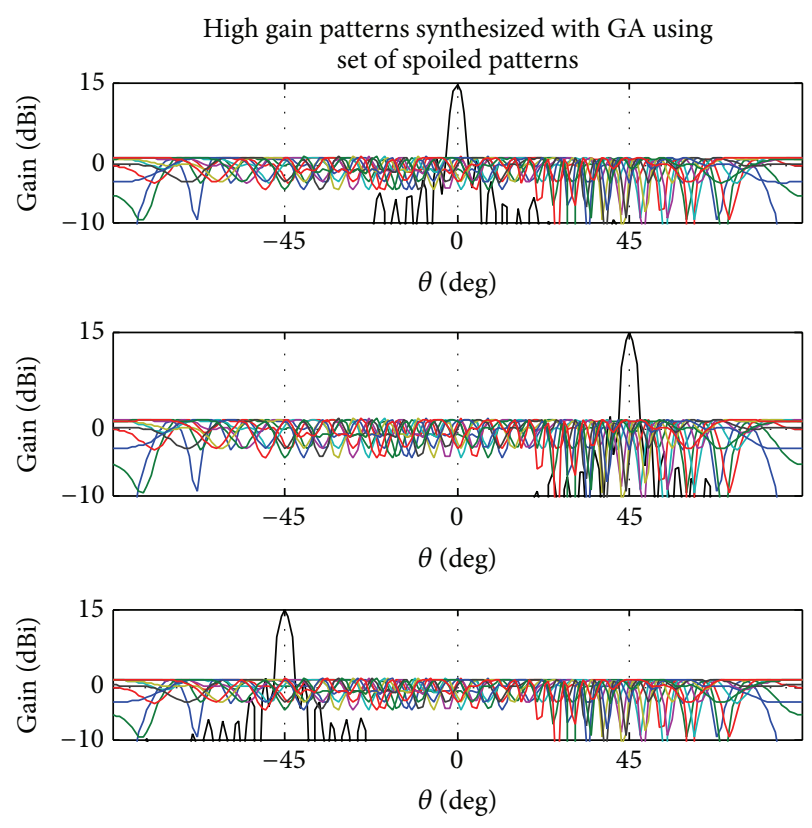

FIGURE 8: Synthesis of high gain beam by GA calculated weights with fixed $N=32$ and $R=8 \mathrm{~km}$.
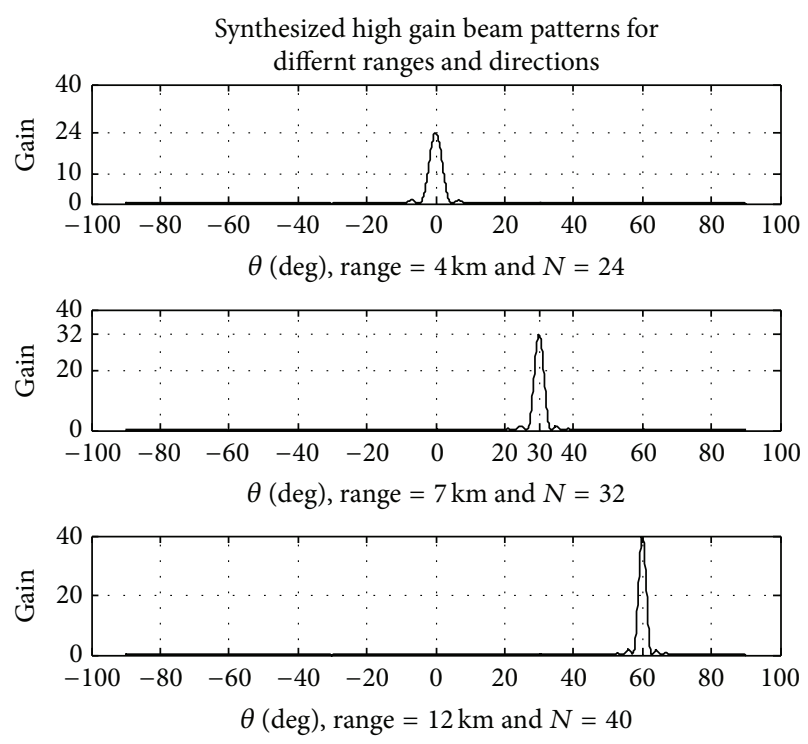

FIGURE 9: Synthesized high gain patterns at different ranges with corresponding array elements.

The proposed hybrid cognitive PAR transmitter receives the estimated target position sent as feedback from the receiver. The transmitter of the proposed radar directs the energy towards that estimated target position keeping in view the range of the target. Proposed radar enjoys the advantages of both the cognitive radar and the PAR radar simultaneously, along with the additional LPI property. 

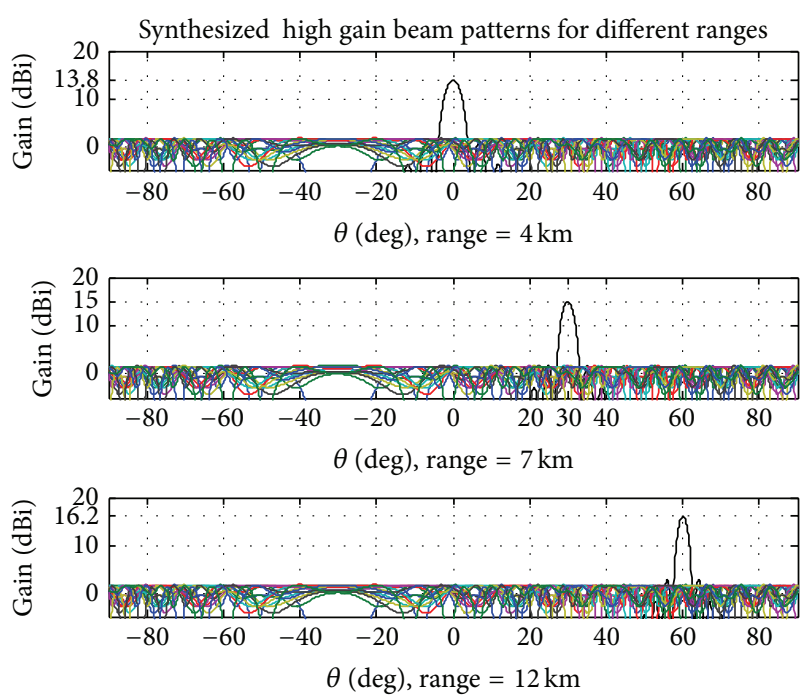

FIGURE 10: Synthesized respective beam patterns with gain in $\mathrm{dBi}$.

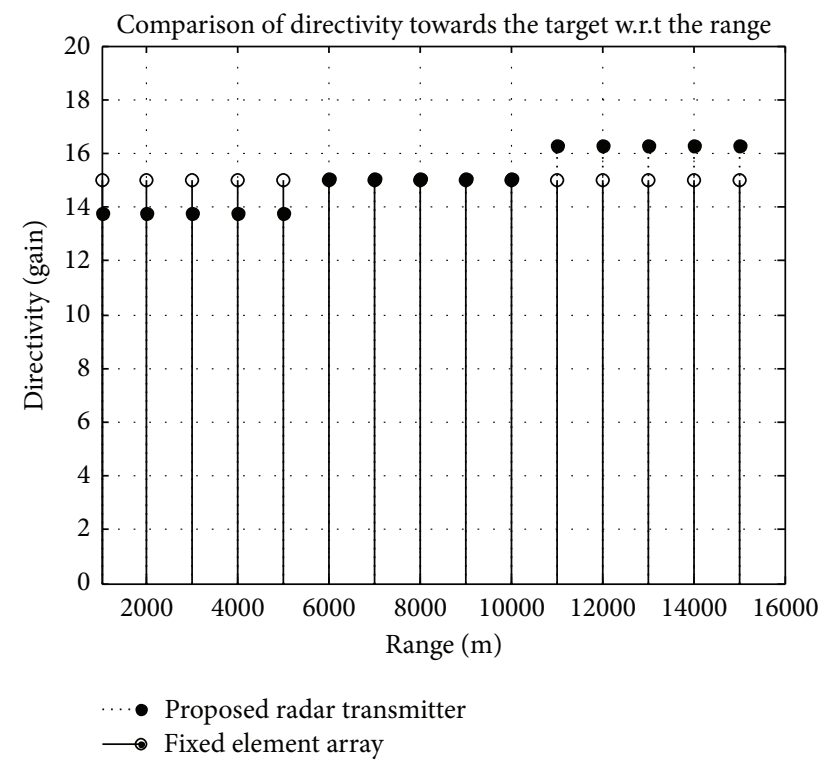

FIGURE 11: Comparison of incident power on the target with respect to the range.

\section{Conflict of Interests}

The authors declare that there is no conflict of interests regarding the publication of this paper.

\section{References}

[1] X. Zhang, X. Gao, and Z. Wang, "Blind PARALIND multiuser detection for smart antenna CDMA system over multipath fading channel," Progress in Electromagnetics Research, vol. 89, pp. 23-38, 2009.

[2] Y. Xue, Cognitive radar: theory and simulations [Ph.D. thesis], School of Graduate Studies, McMaster University, Ontario, Canada, 2010.
[3] S. Haykin, "Cognitive radar," IEEE Signal Processing Magazine, vol. 23, no. 1, pp. 30-40, 2006.

[4] S. Haykin, Y. Xue, and P. Setoodeh, "Cognitive radar: step toward bridging the gap between neuroscience and engineering," Proceedings of the IEEE, vol. 100, no. 11, pp. 3102-3130, 2012.

[5] A. Hassanien and S. A. Vorobyov, "Phased-MIMO radar: a tradeoff between phased-array and MIMO radars," IEEE Transactions on Signal Processing, vol. 58, no. 6, pp. 3137-3151, 2010.

[6] M. Mouhamadou, P. Vaudon, and M. Rammal, "Smart antenna array patterns synthesis: null steering and multi-user beamforming by phase control," Progress in Electromagnetics Research, vol. 60, pp. 95-106, 2006.

[7] D. E. Lawrence, "Low probability of intercept antenna array beamforming," IEEE Transactions on Antennas and Propagation, vol. 58, no. 9, pp. 2858-2865, 2010.

[8] D. C. Schleher, Introduction to Electronic Warfare, Artech House, Boston, Mass, USA, 1986.

[9] K. L. Fuller, “To see and not be seen [radar]," IEE Proceedings, Part F: Communications, Radar and Signal Processing, vol. 137, no. 1, pp. 1-10, 1990.

[10] E. J. Carlson, "Low probability of intercept (LPI) techniques and implementations for radar systems," in Proceedings of the 1988 IEEE National Radar Conference, pp. 56-60, April 1988.

[11] P. E. Pace, Detecting and Classifying Low Probability of Intercept Radar, Artech House, Norwood, Mass, USA, 2004.

[12] D. C. Schleher, "LPI radar: fact or fiction," IEEE Aerospace and Electronic Systems Magazine, vol. 21, no. 5, pp. 3-6, 2006.

[13] F. zaman, I. M. Qureshi, A. Naveed, J. A. khan, and R. M. A. Zahoor, "Amplitude and Directional of Arrival estimation: comparison between different techniques," Progress in Electromagnetics Research B, vol. 39, pp. 319-335, 2012.

[14] J. Gavan and J. S. Ishay, "Hypothesis of natural radar tracking and communication direction finding systems affecting hornets flight," Journal of Electromagnetic Waves and Applications, vol. 16, no. 2, pp. 247-248, 2002.

[15] Z. Fawad, I. M. Qureshi, A. N. Malik, and Z. U. Khan, "Joint amplitude: range and direction of arrival estimation of near field sources using hybrid differential evolution and hybrid particle swarm intelligence," Archives des Sciences, vol. 65, pp. 671-685, 2012.

[16] N. J. Gordon, D. J. Salmond, and A. F. M. Smith, "Novel approach to nonlinear/non-gaussian Bayesian state estimation," IEE Proceedings, Part F: Radar and Signal Processing, vol. 140, no. 2, pp. 107-113, 1993.

[17] F. H. Lewis, Optical Estimation with an Introduction to Stochastic Control Theory, Wiley, New York, NY, USA, 1986.

[18] M. S. Grewal and A. P. Andrews, Kalman Filtering: Theory and Practice, Prentice-Hall, Englewood Cliffs, NJ, USA, 1993.

[19] H. L. van Trees, Detection, Estimation, and Modulation Theory, part 1, Wiley, New York, NY, USA, 1968.

[20] Y. Bar-Shalom, X. Rong Li, and T. Kirubarajan, Estimation with Application to Tracking and Navigation, John Wiley \& Sons, 2004.

[21] Y. Bresler, "Two filter formulae for discrete time nonlinear Bayesian smoothing," International Journal of Control, vol. 43, no. 2, pp. 629-641, 1986.

[22] Y. Bar-shalom and K. Birmiwal, "Variable dimension filter for maneuvering target tracking," IEEE Transactions on Aerospace and Electronic Systems, vol. 18, no. 5, pp. 621-629, 1982. 
[23] S. Michael Herman, A particle filtering approach to joint passive radar tracking and target classification [Ph.D. thesis], University of Illinois at Urbana-Champaign, 2002.

[24] R. E. Kalman, "A new approach to linear filtering and prediction problems," Transactions of the ASME, Series D: Journal of Basic Engineering, vol. 82, pp. 34-45, 1960.

[25] R. Hecht-Nielsen, "Replicator neural networks for universal optimal source coding," Science, vol. 269, no. 5232, pp. 18601863, 1995.

[26] S. Haykin, Neural Networks and Learning Machines, PrenticeHall, Englewood Cliffs, NJ, USA, 3rd edition, 2009. 

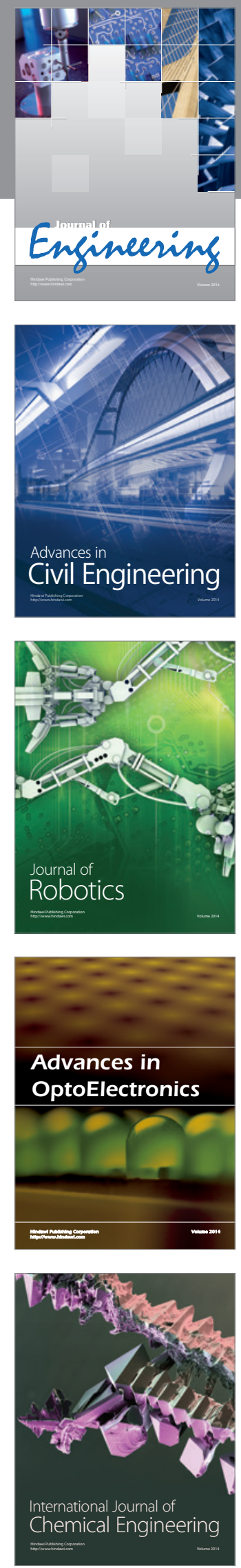

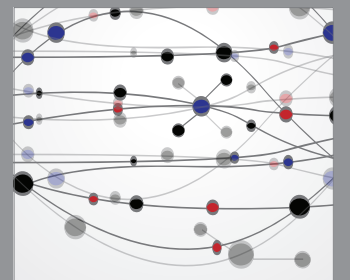

The Scientific World Journal
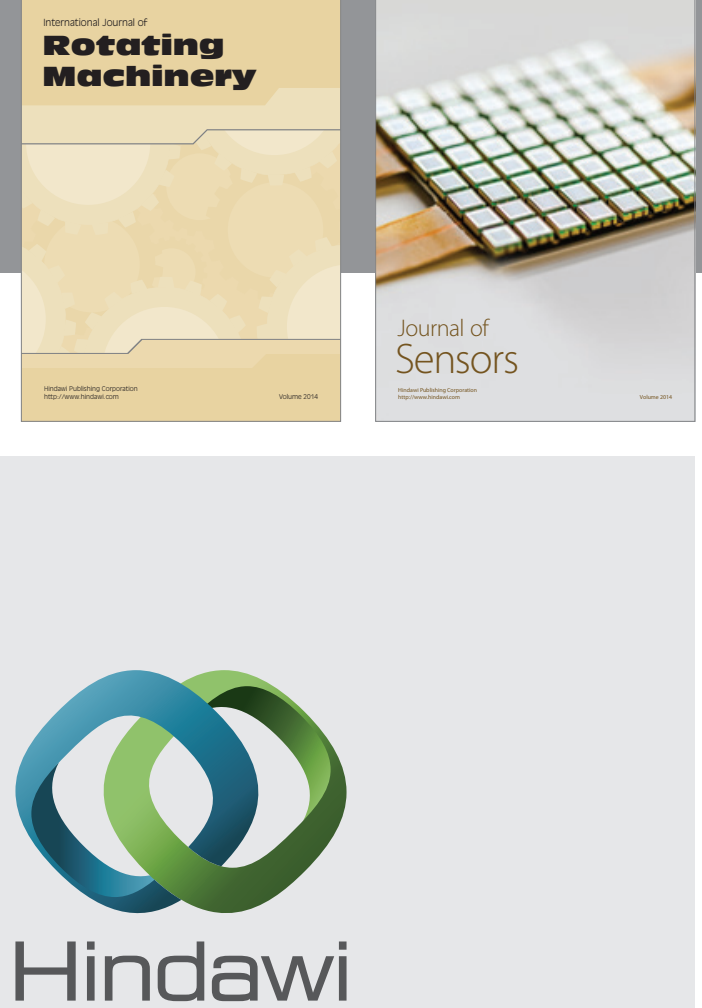

Submit your manuscripts at http://www.hindawi.com
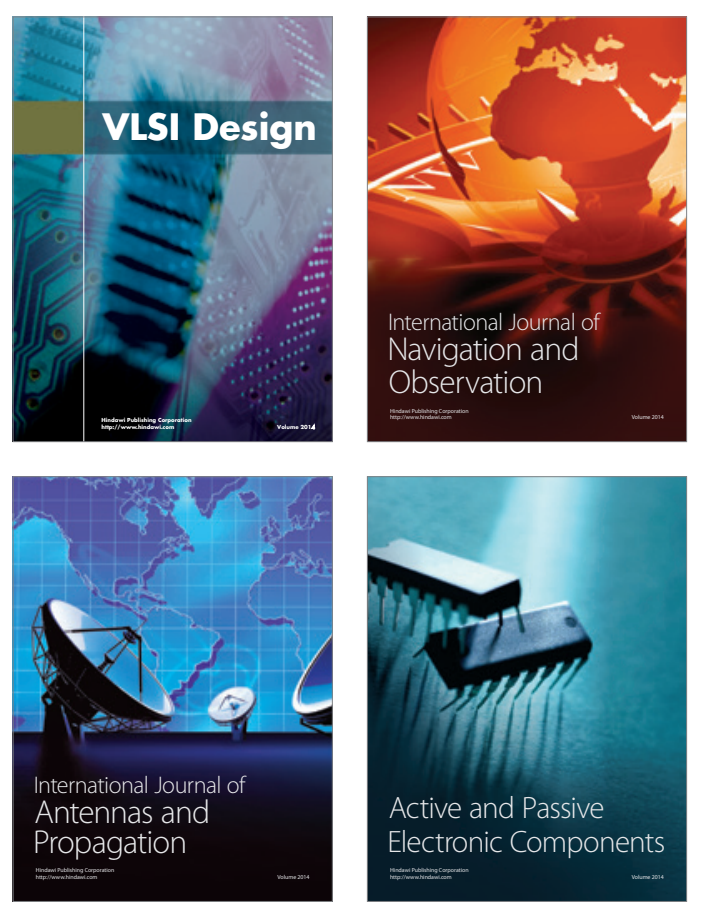
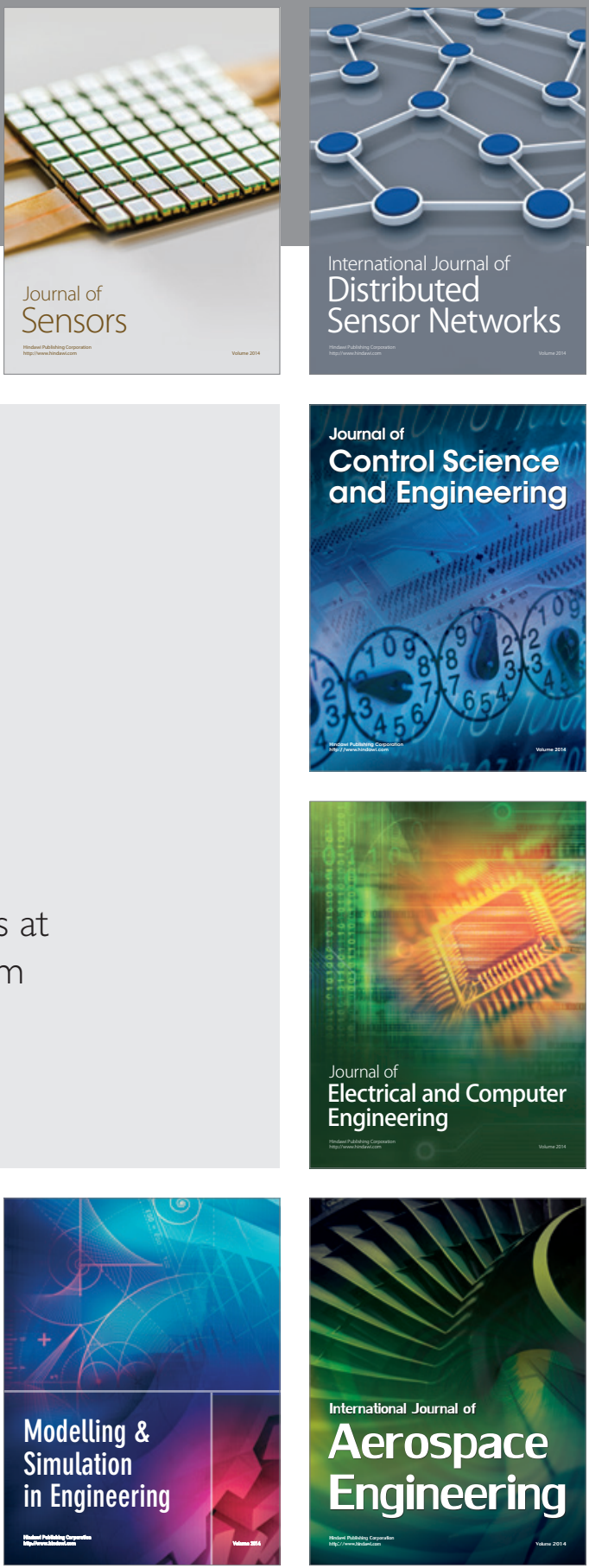

Journal of

Control Science

and Engineering
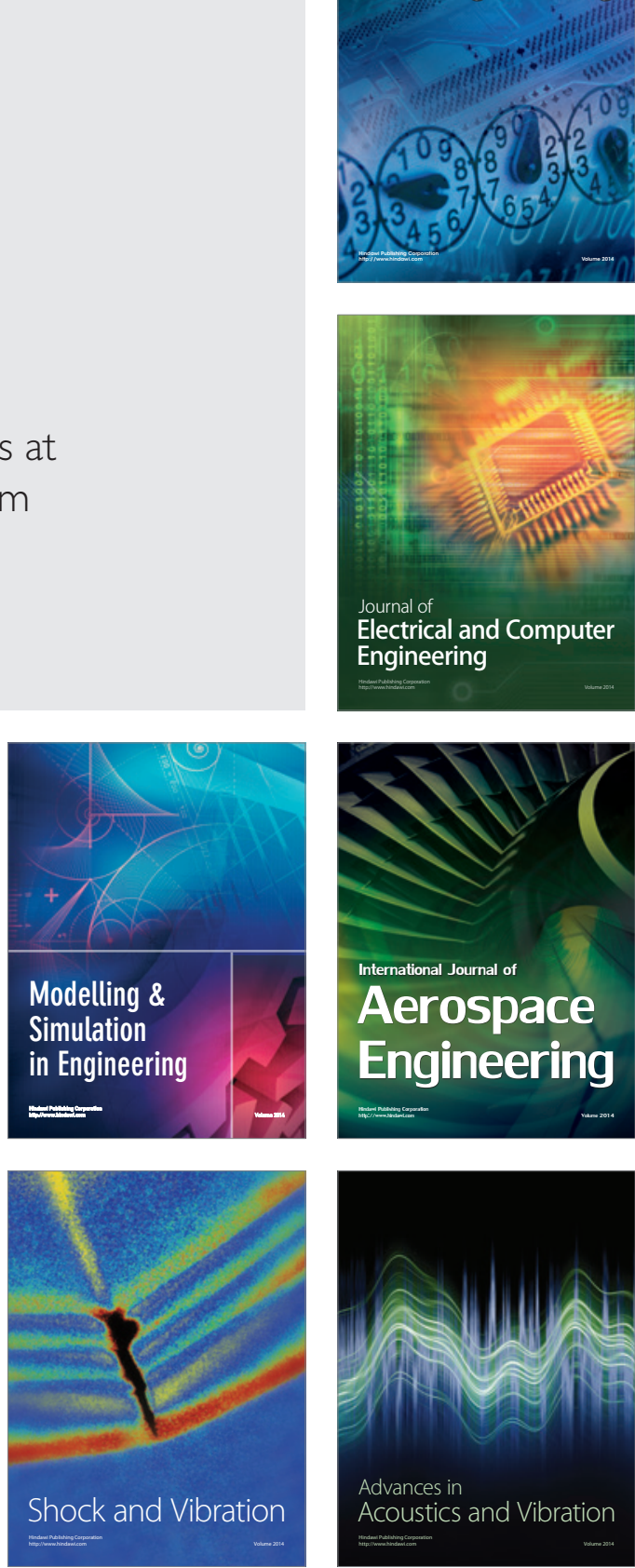